\title{
Susceptibility to viral and mycobacterial infections
}

INSERM

\section{Source}

INSERM. (1999). Orphanet: an online rare disease and orphan drug data base.

Susceptibility to viral and mycobacterial infections. ORPHA:391311

Susceptibility to viral and mycobacterial infections is a rare, genetic, primary immunodeficiency due to a defect in innate immunity disorder characterized by impaired intracellular signaling from both type I and type II interferons, leading to early-onset, severe, life-threatening intracellular bacterial (typically mycobacteria) and viral (mainly herpes viruses) infections. 